\title{
Spatial pattern formation and delay induced destabilization in predator-prey model with fear effect
}

\author{
Swati Mishra ${ }^{1}$ and RANJIT UPADHYY ${ }^{1}$ \\ ${ }^{1}$ Indian Institute of Technology (Indian School of Mines) Dhanbad
}

May 22, 2021

\begin{abstract}
Recent field experiments showed that predators influence the prey population not only by direct consumption but also by stimulating various defensive strategies. The cost of these defensive strategies can include energetic investment in defensive structures, reduced energy income, lower mating success, and emigration which ultimately reduces the reproduction of prey. To explore the effect of these defensive strategies (anti-predator behaviors), a modified Leslie-Gower predator-prey model with the cost of fear has been considered. Gestation delay is also incorporated in the system for a more realistic formulation. Boundedness, equilibria and stability analysis of the temporal model are studied. By considering gestation delay as a bifurcation parameter, the existence of Hopf-bifurcation around the interior equilibrium point is discussed together with the direction, stability and period of bifurcating solutions arising through Hopf-bifurcation. The spatial extension of the proposed model incorporating density-dependent cross-diffusion is also investigated and the conditions for diffusion-driven instability are obtained. To illustrate the analytical findings, detailed numerical simulations are performed. Biologically realistic Turing patterns as hexagonal spots, spots and stripes mixture, and labyrinthine type patterns are identified. It is found that the fear level has a stabilizing impact on delay induced destabilization and both stabilizing and destabilizing effects on Turing instability.
\end{abstract}

\section{Hosted file}

Manuscript.pdf available at https://authorea.com/users/415177/articles/523067-spatialpattern-formation-and-delay-induced-destabilization-in-predator-prey-model-with-feareffect 